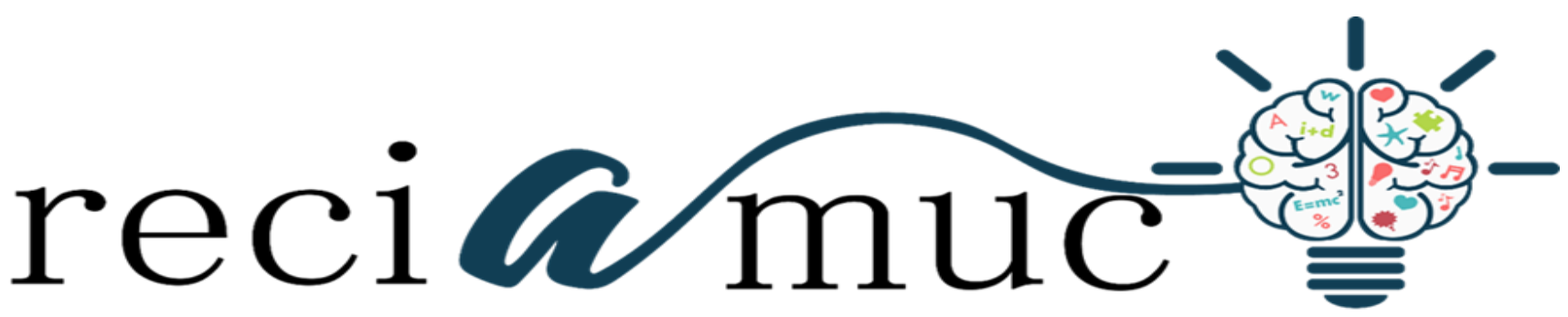

Revista cientifica de investigación actualización del mundo de las ciencias

Marcelo Kevin Taiano Espinoza a, William Aarón Vargas Samerón ${ }^{\text {b; }}$ Luis Carlos

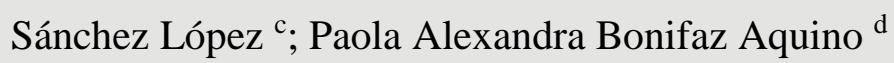

Diagnóstico diferencial de la disfagia

Differential diagnosis of dysphagia

Revista Científica de Investigación actualización del mundo de las Ciencias. Vol. 3 núm., 1, enero, ISSN: 2588-0748, 2018, pp. 587-617

DOI: $10.26820 /$ reciamuc/3.(1).enero.2019.587-617

URL: $\underline{\text { http://reciamuc.com/index.php/RECIAMUC/article/view/249 }}$

Código UNESCO: 3205 Medicina Interna

Tipo de Investigación: Artículo de Revisión

Editorial Saberes del Conocimiento

Recibido: 10/12/2018

Aceptado: 20/01/2019

Publicado: 30/01/2019

Correspondencia: director@reciamuc.com

a. Médico; Guayaquil, Ecuador.

b. Médico; Guayaquil, Ecuador.

c. Médico; Guayaquil, Ecuador.

d. Médico; Guayaquil, Ecuador. 


\section{Diagnóstico diferencial de la disfagia}

Vol. 3, núm. 1., (2019)

Marcelo Kevin Taiano Espinoza; William Aarón Vargas Samerón; Luis Carlos, Sánchez López;

Paola Alexandra Bonifaz Aquino

\section{RESUMEN}

El organismo necesita mantener un equilibrio bioquímico y físico que permita el normal desarrollo de las actividades del individuo. La energía interna es manifestada a través de la alimentación que digiere la persona. La deglución consiste en trasportar el alimento desde la boca hasta el estómago. Cuando existe un problema dentro de los órganos que conforman este mecanismo se produce una disfagia, la cual dificulta mover, formar y trasportar el bolo de alimentación. Por lo cual, el objetivo general de esta investigación es analizar el diagnóstico diferencial de la disfagia. La metodología que se utilizó fue basada en investigaciones documentales y bibliográficas. Como resultado se analizó las características de la disfagia entre ella sus tipos, síntomas y causas, así como también determinar el diagnóstico diferencial de esta enfermedad. Como conclusión, para determinar el diagnóstico diferencial de la disfagia se requiere del historial del paciente, del test clínico que ayudara a conocer los síntomas que padece y del examen físico que se realiza en primer momento.

Palabras Claves: Deglución; Disfagia; Síntomas; Causas; Diagnóstico Diferencial. 


\title{
Diagnóstico diferencial de la disfagia
}

Vol. 3, núm. 1., (2019)

Marcelo Kevin Taiano Espinoza; William Aarón Vargas Samerón; Luis Carlos, Sánchez López;

Paola Alexandra Bonifaz Aquino

\begin{abstract}
The organism needs to maintain a biochemical and physical balance that allows the normal development of the individual's activities. The internal energy is manifested through the food that the person digests. Swallowing involves transporting food from the mouth to the stomach. When there is a problem within the organs that make up this mechanism, dysphagia occurs, which makes it difficult to move, form and transport the bolus of food. Therefore, the general objective of this research is to analyze the differential diagnosis of dysphagia. The methodology used was based on documentary and bibliographic research. As a result, we analyzed the characteristics of dysphagia among them, their types, symptoms and causes, as well as determining the differential diagnosis of this disease. In conclusion, to determine the differential diagnosis of dysphagia requires the patient's history, the clinical test that will help to know the symptoms he suffers and the physical examination that is performed in the first moment.
\end{abstract}

Key Words: Swallowing Dysphagia Symptom; Causes; Differential Diagnosis. 


\section{Diagnóstico diferencial de la disfagia}

Vol. 3, núm. 1., (2019)

Marcelo Kevin Taiano Espinoza; William Aarón Vargas Samerón; Luis Carlos, Sánchez López; Paola Alexandra Bonifaz Aquino

\section{Introducción.}

El cuerpo humano debe tener las energías necesarias para poder realizar distintas tareas diarias necesarias para su desarrollo. Para cumplir con esto se debe buscar el ingreso de las energías internas que permitan tal funcionamiento y con ello poseer un equilibrio en todo el sistema. Estas energías internas provienen en su mayoría de una sana y buena alimentación. Uno de los aparatos que permite la introducción de estos alimentos es el esófago. Dentro del organismo existen procesos físicos y químicos que transforman estos alimentos en la energía necesaria para realizar esas tareas diarias. Por lo cual es importante conocer la importancia de la deglución.

La deglución es una acción motora en la que actúan múltiples órganos y músculos para la transformación de los alimentos en el estómago. La deglución es un proceso neuromuscular complejo, mediante el cual los alimentos procedentes de la boca pasan por la faringe y esófago al estómago, a través de la acción motora automática del bolo alimenticio hacia el esófago(Vargas, Navas, \& Medina, 2016). Es un proceso complejo de integración sensorial y motora, la regulación y el control cerebral de múltiples estructuras anatómicas músculos nervios y una estrecha coordinación del sistema respiratorio y del sistema nervioso central (Vilardell Navarro, 2017).

La deglución se produce gracias a las estructuras anatómicas como es la boca y la lengua, la faringe, la laringe y el esófago. La boca corresponde al vestíbulo de la lengua y cavidad oral. El vestíbulo de la boca se sitúa por delante de los dientes, y queda delimitado externamente por las mejillas y los labios, y la lengua es un órgano musculoso que participa en la succión, la 


\section{Diagnóstico diferencial de la disfagia}

Vol. 3, núm. 1., (2019)

Marcelo Kevin Taiano Espinoza; William Aarón Vargas Samerón; Luis Carlos, Sánchez López;

Paola Alexandra Bonifaz Aquino

masticación, la deglución y la fonación (Vilardell Navarro, 2017). En la Figura 1 se puede observar los músculos suprahioideos de la mandíbula y del hueso hioides.

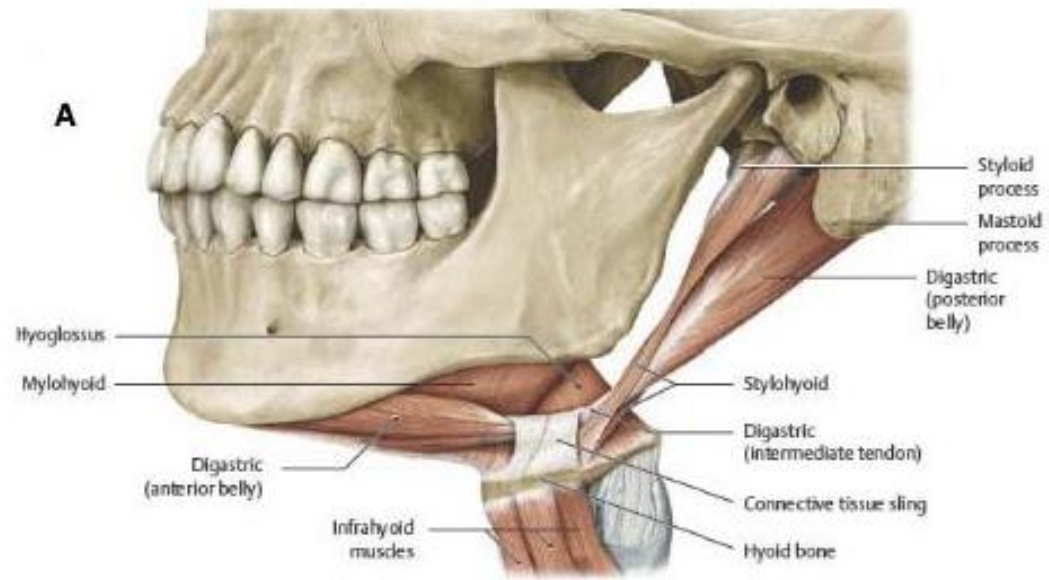

B

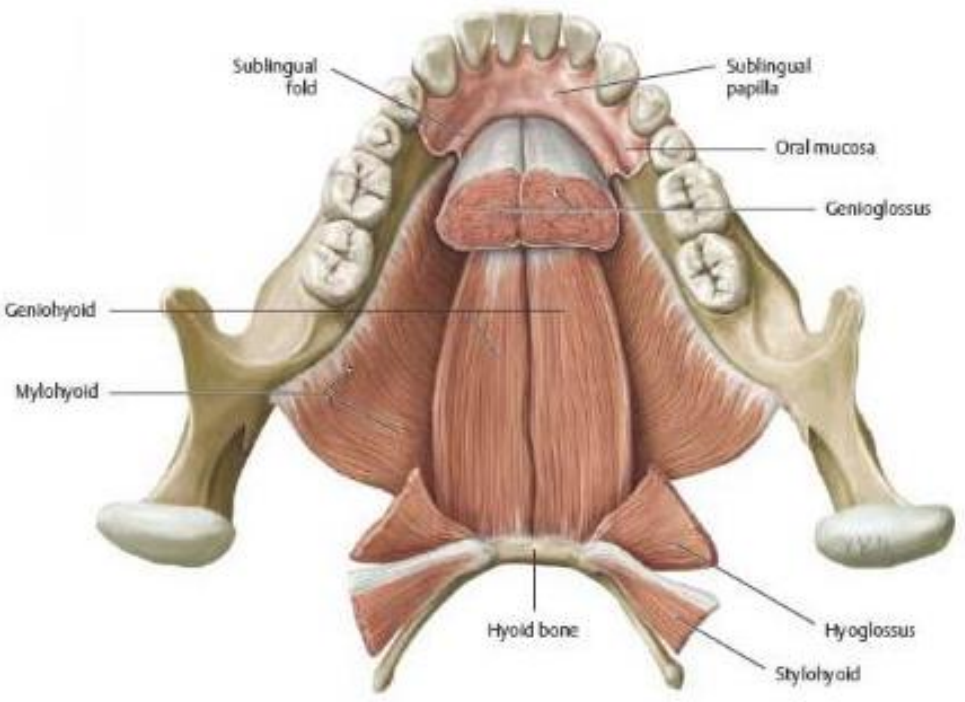

Figura 1. Músculos suprahioideos, (A) vista lateral y (B) vista superior, de la mandíbula y del hueso hioides

Fuente: (Vilardell Navarro, 2017) 


\section{Diagnóstico diferencial de la disfagia}

Vol. 3, núm. 1., (2019)

Marcelo Kevin Taiano Espinoza; William Aarón Vargas Samerón; Luis Carlos, Sánchez López; Paola Alexandra Bonifaz Aquino

La importancia de la faringe radica es que forma parte del tubo digestivo para la deglución y la respiración. El extremo superior comunica con las fosas nasales, medialmente con la cavidad oral y caudalmente con la faringe y la tráquea, por una parte, y con el esófago por la otra(Vilardell Navarro, 2017). Esta se puede ver en la Figura 2.

(A)
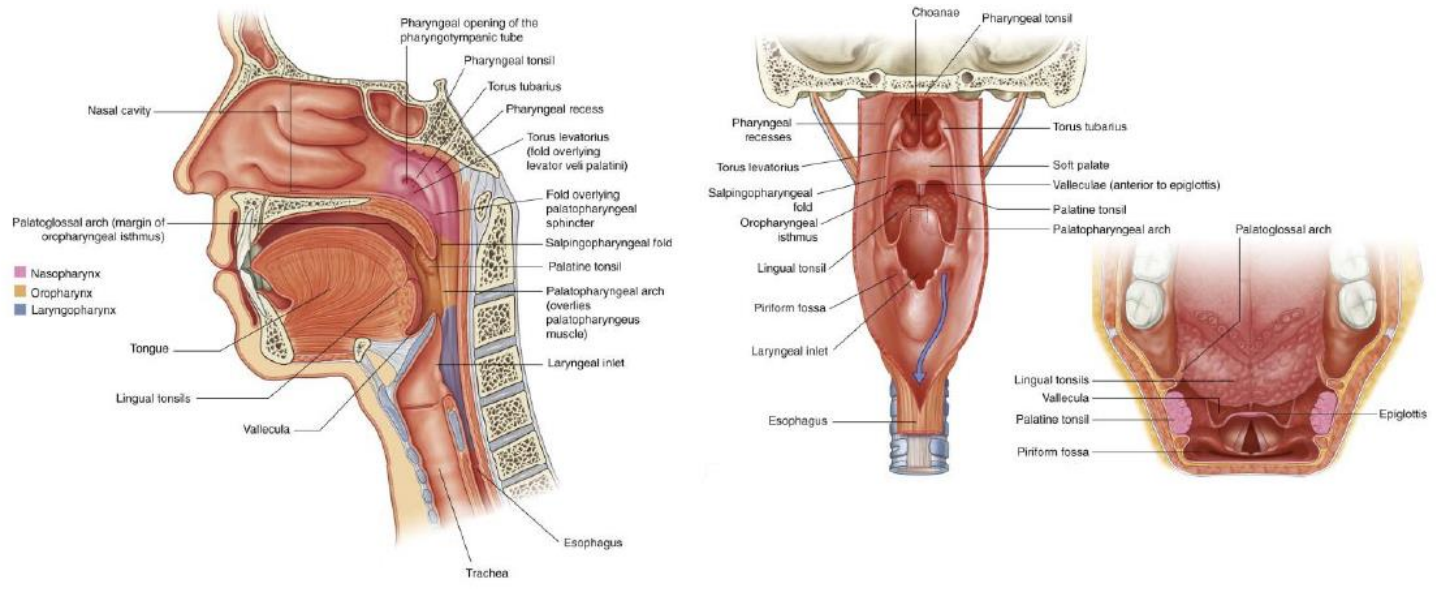

(B)

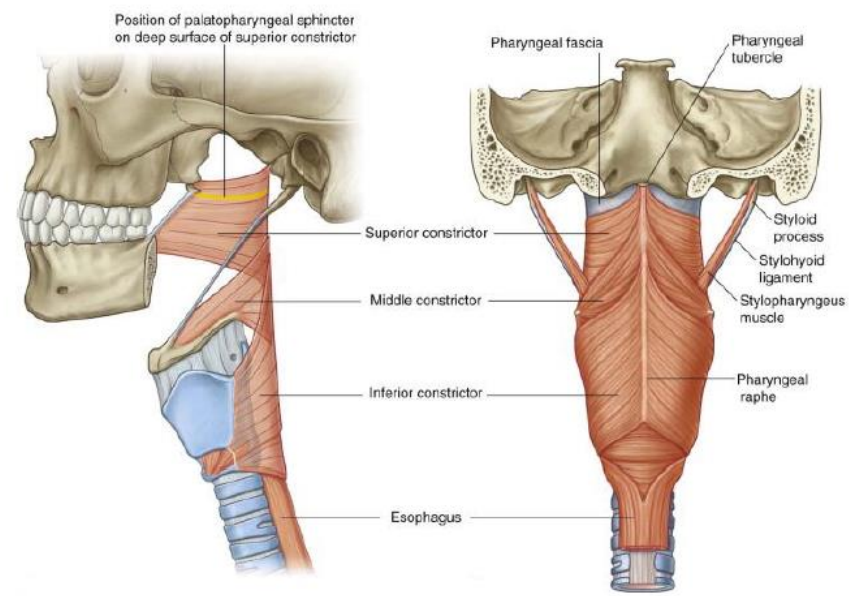

Figura 2. (A) Anatomía y (B) musculatura de la faringe

Fuente: (Vilardell Navarro, 2017) 


\section{Diagnóstico diferencial de la disfagia}

Vol. 3, núm. 1., (2019)

Marcelo Kevin Taiano Espinoza; William Aarón Vargas Samerón; Luis Carlos, Sánchez López;

Paola Alexandra Bonifaz Aquino

Por delante de la faringe se encuentra la laringe situada en la parte media y anterior al cuello. Cranealmente comunica con la cavidad bucal y las fosas nasales, y caudalmente se continúa con la tráquea, donde interviene en la respiración, la deglución y la fonación; y está formada por pequeños músculos responsables de su movimiento (Vilardell Navarro, 2017). En la Figura 3 se detalla las diferentes vistas de la laringe.

La última estructura por la cual depende la deglución es el esófago. Es un tubo fibromuscular, no rectilíneo, flexible y fácilmente dilatable que se extiende desde la laringofaringe hasta el estómago (Vilardell Navarro, 2017).

Por lo cual, el proceso de alimentación es complejo porque interviene la masticación y deglución normal que a la medida que avanza el individuo en edad este proceso afecta su funcionamiento. Son proceso de movimientos voluntarios e involuntarios en el que participan al menos seis pares craneales, los tres primeros segmentos de los nervios cervicales y los 26 músculos de la boca, faringe y esófago(Jiménez Rojas, Corregidor Sánchez, \& Gutiérrez Bezón, 2018). En la Tabla 1 se puede detallar la fisiología de la deglución normal y cambios en la edad. 


\section{Diagnóstico diferencial de la disfagia}

Vol. 3, núm. 1., (2019)

Marcelo Kevin Taiano Espinoza; William Aarón Vargas Samerón; Luis Carlos, Sánchez López; Paola Alexandra Bonifaz Aquino
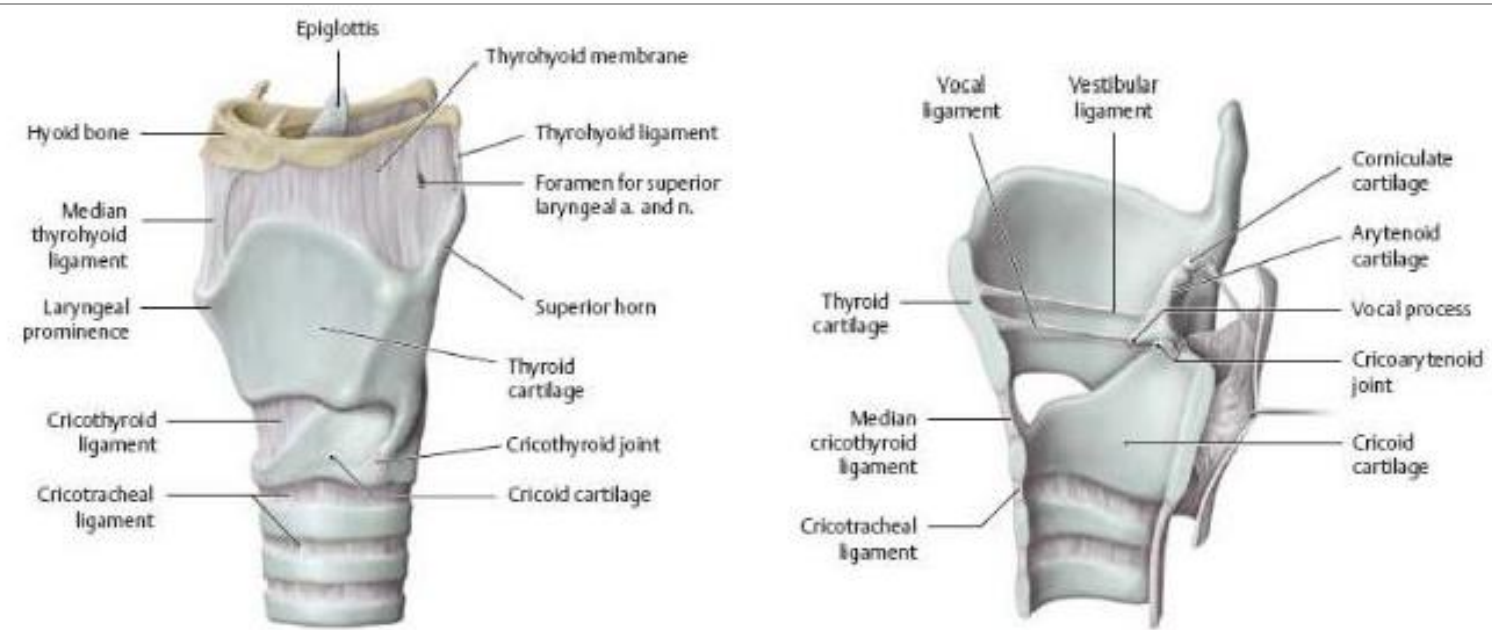

A Left anterior oblique view.

B Sagittal section, viewed from the left medial aspect. The arytenoid cartilage alters the position of the vocal folds during phonation.

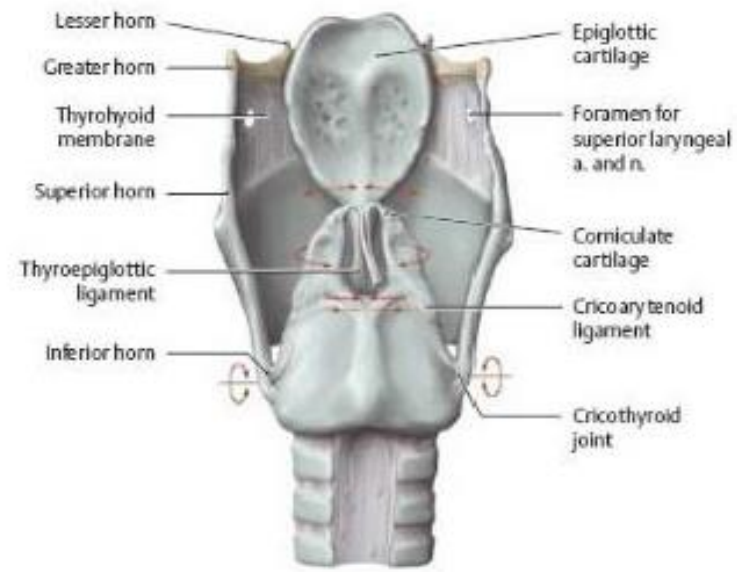

C Posterior view. Arrows indicate the directions of movement in the various joints.

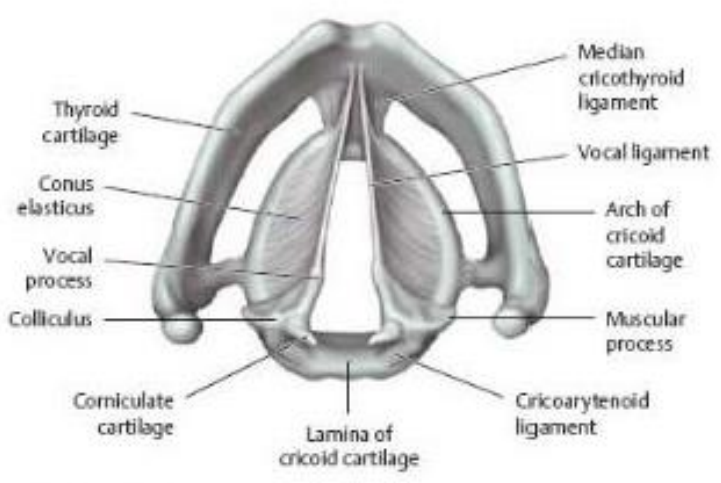

D Superior view.

Figura 3. Las diferentes vistas de la laringe. (A) Vista anterior, /B) vista sagital, (C) vista posterior y $(D)$ vista superior

Fuente: (Vilardell Navarro, 2017) 


\section{Diagnóstico diferencial de la disfagia}

Vol. 3, núm. 1., (2019)

Marcelo Kevin Taiano Espinoza; William Aarón Vargas Samerón; Luis Carlos, Sánchez López;

Paola Alexandra Bonifaz Aquino

\section{Tabla 1. Fisiología de la deglución normal y cambios en la edad}

\begin{tabular}{|c|c|}
\hline Fases de la deglución & Cambios con la edad \\
\hline Fase oral (voluntaria) & \\
\hline $\begin{array}{l}\text { 1. Preparatoria: } \\
\text { - Masticación. } \\
\text { - Formación del bolo. } \\
\text { 2. Tránsito: } \\
\text { - Bolo en base de la lengua. } \\
\text { - Propulsión contra el paladar duro y faringe. } \\
\text { 3. Reflejo deglutorio: } \\
\text { - Estimulación de los pilares amigdalinos e inicio del reflejo. }\end{array}$ & $\begin{array}{l}\text { 1. Pérdida de piezas dentarias. } \\
\text { 2. Desajuste de la prótesis. } \\
\text { 3. Atrofia de los músculos de la masticación. } \\
\text { 4. Disminuye la producción de saliva. }\end{array}$ \\
\hline $\begin{array}{l}\text { Fase faríngea (involuntaria) } \\
\text { 1. Elevación del paladar blando. } \\
\text { 2. El músculo constrictor superior evita la regurgitación. } \\
\text { 3. Peristaltis faríngea hacia hipofaringe. } \\
\text { 4. Cierre de cuerdas vocales. } \\
\text { 5. La laringe se mueve hacia delante y hacia arriba. } \\
\text { 6. La epiglotis cae sobre la apertura laríngea. } \\
\text { 7. Apertura del esfínter cricofaríngeo. }\end{array}$ & $\begin{array}{l}\text { 1. Disminuye el tono muscular que puede alterar el } \\
\text { aclaramiento faríngeo. } \\
\text { 2. Mayor propensión al desarrollo de divertículos. } \\
\text { 3. Epiglotis más lenta y pequeña. } \\
\text { 4. Alargamiento del tiempo de apnea. }\end{array}$ \\
\hline $\begin{array}{l}\text { Fase esofágica (involuntaria) } \\
\text { 1. Peristaltismo hacia la región esofágica. } \\
\text { 2. Relajación del esfinter esofágico inferior. }\end{array}$ & $\begin{array}{l}\text { Disminuye la amplitud de las contracciones } \\
\text { peristálticas esofágicas. }\end{array}$ \\
\hline
\end{tabular}

Fuente: (Jiménez Rojas, Corregidor Sánchez, \& Gutiérrez Bezón, 2018)

El proceso de deglución se lleva en 4 etapas o fases como lo son la preparación oral, transferencia oral (de manera voluntaria), transferencia faríngea y transferencia esofágica (fase involuntaria) (HUAYTA, 2018). La Figura 4 se puede observar las etapas o fases de la deglución. Estas tienen mecanismos o válvulas que sellan el compartimiento en el cual se desarrollan; así, cuando termina una etapa, se abren estos mecanismos permitiendo al bolo alimenticio pasar a la siguiente etapa(Epprecht González, Trobat Company, \& Novas Valiente, 2006). En la Figura 5 se detalla las distintas fases con sus sellos y compuertas. 


\section{Diagnóstico diferencial de la disfagia}

Vol. 3, núm. 1., (2019)

Marcelo Kevin Taiano Espinoza; William Aarón Vargas Samerón; Luis Carlos, Sánchez López;

Paola Alexandra Bonifaz Aquino

Existe un control neural de la deglución la cual provienen de un proceso entre los nervios craneales, periférico y centrales. Estos llegan hasta los centros deglutorios a nivel del tronco encefálico, donde se produce la integración y se genera la respuesta motora, que finalmente es trasmitida por las motoneuronas de diversos pares craneales hasta la musculatura del tracto digestivo implicada en la deglución (Vilardell Navarro, 2017). Estos procesos complejos y coordenados se visualizan en la Figura 6.
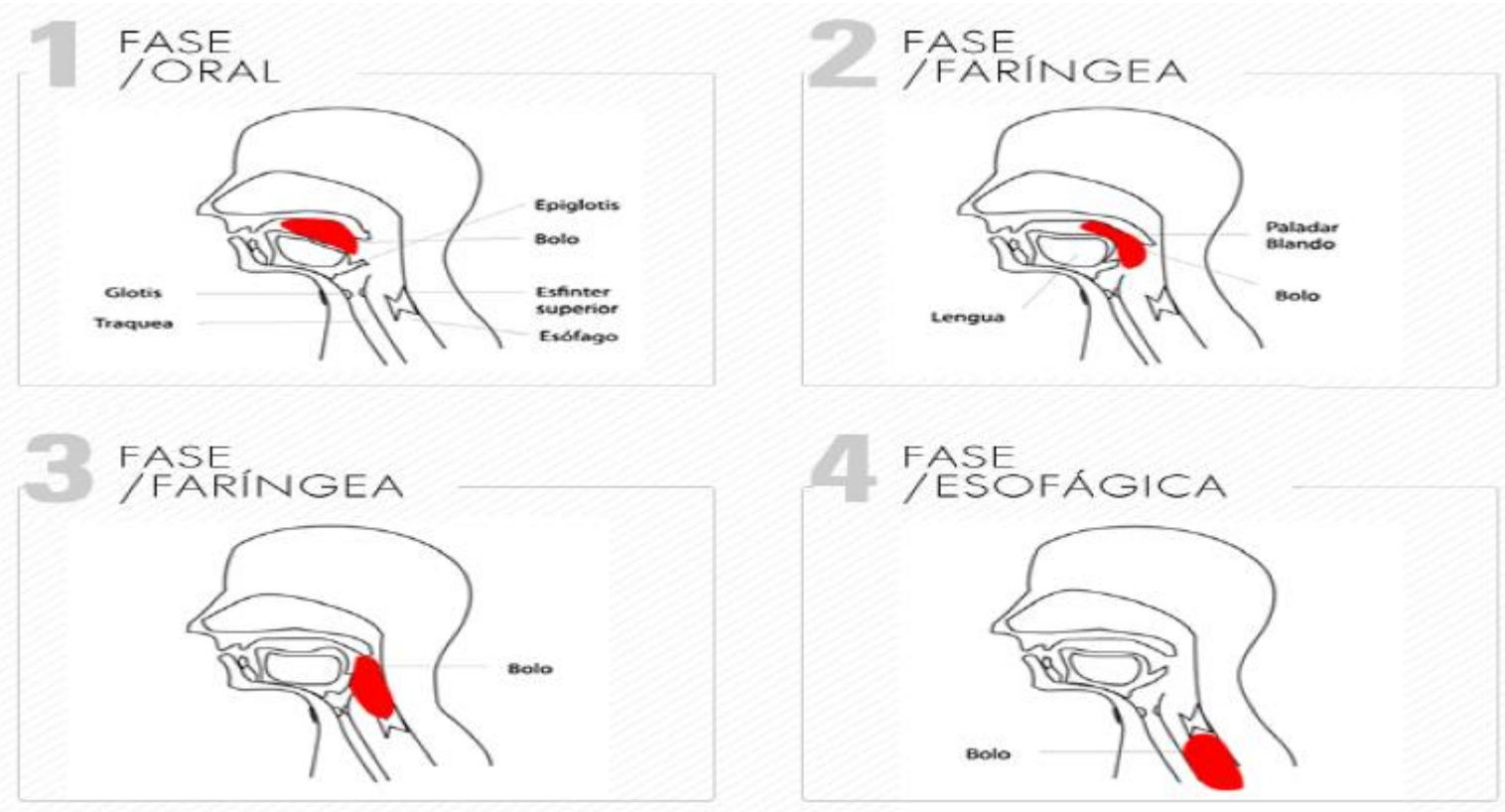

Figura 4.- Fases de la deglución

Fuente: (HUAYTA, 2018) 


\section{Diagnóstico diferencial de la disfagia}

Vol. 3, núm. 1., (2019)

Marcelo Kevin Taiano Espinoza; William Aarón Vargas Samerón; Luis Carlos, Sánchez López;

Paola Alexandra Bonifaz Aquino

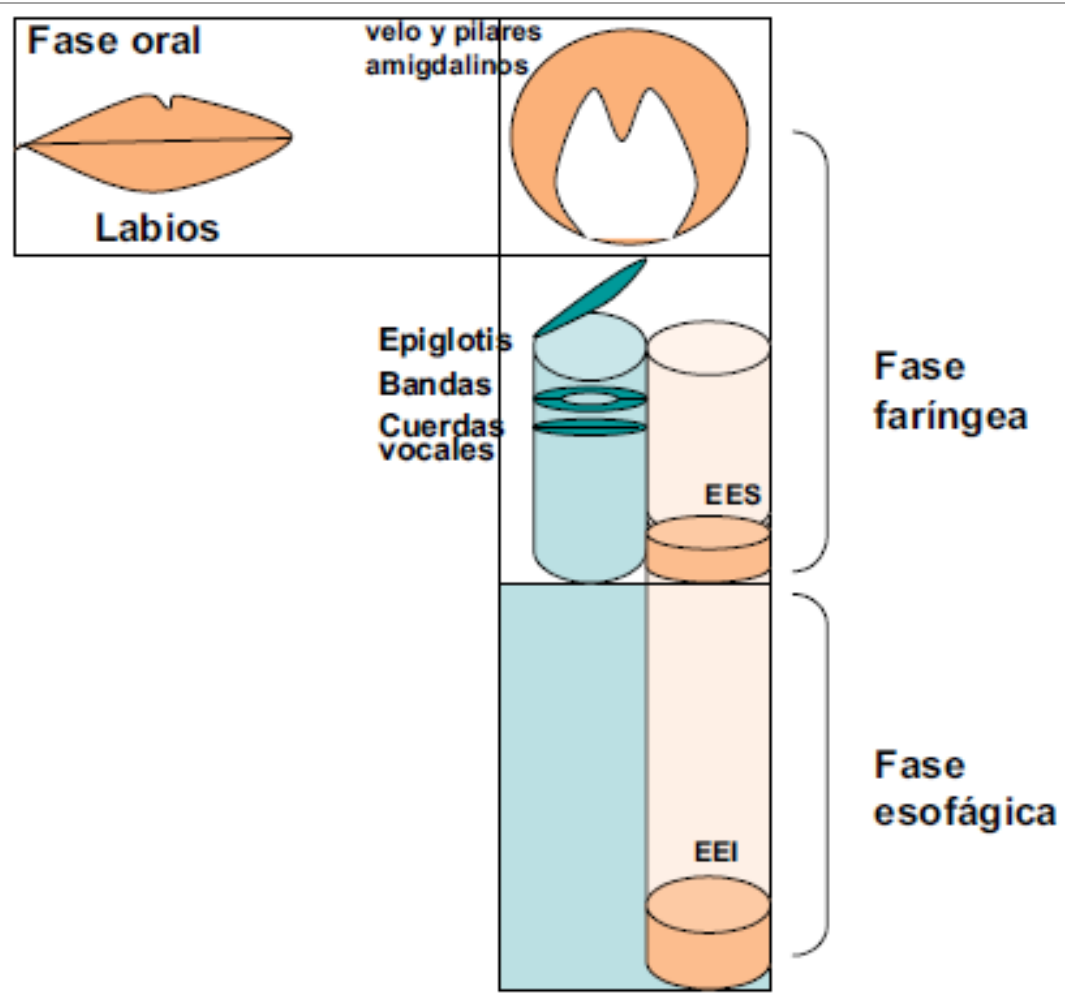

Figura 5. Las distintas fases con sus respectivos sellos o compuertas. Fase oral: labios, velo palatino y pilares amigdalinos. Fase faríngea: velo paladar, epiglotis, bandas y cuerdas vocales, esfinter esofágico superior (EES). Fase esofágica: esfínter esofágico superior e inferior (EEI)

Fuente: (Epprecht González, Trobat Company, \& Novas Valiente, 2006) 


\section{Diagnóstico diferencial de la disfagia}

Vol. 3, núm. 1., (2019)

Marcelo Kevin Taiano Espinoza; William Aarón Vargas Samerón; Luis Carlos, Sánchez López;

Paola Alexandra Bonifaz Aquino

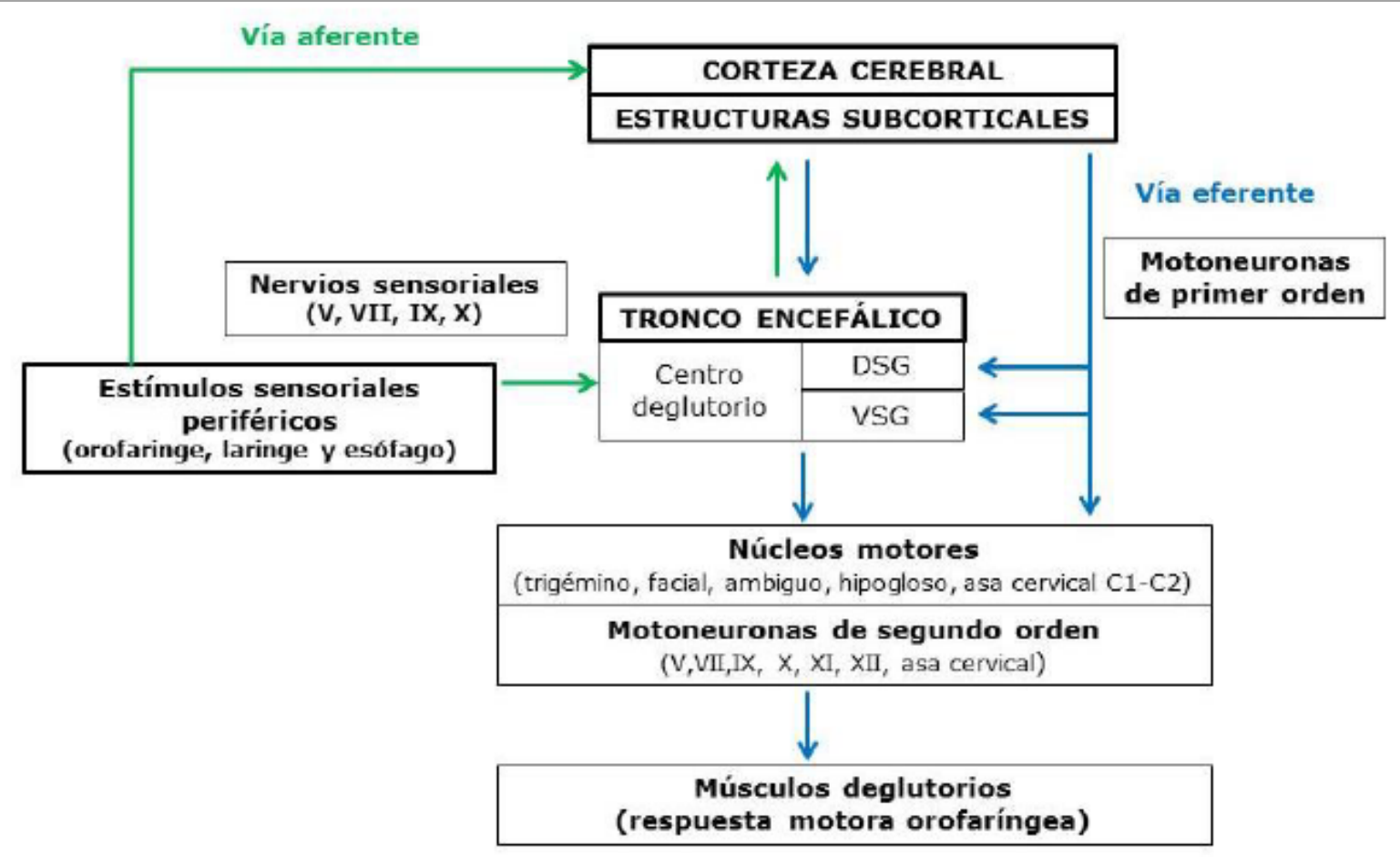

Figura 6. Diagrama del control neural de la deglución

Fuente: (Vilardell Navarro, 2017)

El proceso de deglución también puede tener lesiones que pueden existir en los diferentes niveles. Estos también se pueden detallar en la Tabla 2 que corresponde a la clasificación de los trastornos de la deglución.

$\checkmark \quad$ La alteración en el cierre del paladar predispondrá al reflujo de alimentos hacia la rinofaringe, sobre todo al comer grandes bocados o cambiar de posición (poner la cabeza hacia abajo, no muy habitual mientras e come).

$\checkmark \quad$ La lesión unilateral de peristaltismo faríngeo acumulará alimentos en el lado lesionado y, si es bilateral, tenderá a acumularse en las valéculas, senos piriformeso por toda la faringe. 


\section{Diagnóstico diferencial de la disfagia}

Vol. 3, núm. 1., (2019)

Marcelo Kevin Taiano Espinoza; William Aarón Vargas Samerón; Luis Carlos, Sánchez López;

Paola Alexandra Bonifaz Aquino

Esto predispondrá a la aspiraciónporque al terminar la deglución quedaránrestos de comida en la faringe y al inspirar tenderána caer en la vía aérea.

$\checkmark$ Si existe dificultad para la elevación de la laringe sedificultará el cierre epiglótico favoreciendo la entrada de material residual al vestíbulo laríngeo y potencialmentea la vía aérea en la inhalación siguiente.

$\checkmark \quad$ La reducción o imposibilidad del cierre de la laringeen sus distintos niveles predispondrá a la aspiración por entrada directa de material alimentario.

$\checkmark \quad$ La disfunción de la apertura cricofaríngea de manera aislada es rara; como la elevación de la laringe influyedirectamente en la apertura cricofaríngea es más habitual que se relacione con la dificultad de la motilidadlaríngea. Si hay disfunción cricofaríngea los residuos acumulados a nivel del seno piriforme pueden rebosar hacia las vías respiratorias después de la deglución y aspirarse hacia ellas.(Epprecht González, Trobat Company, \& Novas Valiente, 2006, pág. 68) 


\section{Diagnóstico diferencial de la disfagia}

Vol. 3, núm. 1., (2019)

Marcelo Kevin Taiano Espinoza; William Aarón Vargas Samerón; Luis Carlos, Sánchez López; Paola Alexandra Bonifaz Aquino

\section{Tabla 2. Clasificación de los trastornos de la deglución}

\begin{tabular}{|c|c|}
\hline FISIOLOGLA DE LA FASE ORAL & DISFUNCIÓN ORAL \\
\hline $\begin{array}{l}\text { - Cierre de los labios. } \\
\text { - Preparación de texturas. } \\
\text { - Movimientos de la lengua. } \\
\text { - Masticación. } \\
\text { - Transporte del bolo. } \\
\text { - Cierre en la cavidad nasal. }\end{array}$ & $\begin{array}{l}\text { - Aspiración previa a la deglución faríngea. } \\
\text { - Fuga (anterior, posterior). } \\
\text { - Residuo oral. } \\
\text { - Movimientos ineficaces de la lengua y para la masticación. } \\
\text { - Transporte tardio del bolo. } \\
\text { - Distribución difusa del bolo en la cavidad oral. } \\
\text { - Propulsión lingual de los alimentos y los líquidos. } \\
\text { - Regurgitación hacia la cavidad nasal. }\end{array}$ \\
\hline FISIOLOGIA DE LA FASE FARINNGEA & DISFUNCION FARINGEA \\
\hline $\begin{array}{l}\text { - Elevación de la laringe. } \\
\text { - Movimiento del hueso hioides. } \\
\text { - Inclinación de la epiglotis. } \\
\text { - Cierre del vestíbulo de la laringe. } \\
\text {-Cierre del vestibulo de la laringe: } \\
\text { penetración/aspiración. } \\
\text { - Actividad de los músculos constrictores: } \\
\text { retención faringea. } \\
\text { - Apertura del segmento faringoesofágico (SFE). }\end{array}$ & $\begin{array}{l}\text { - Aspiración durante la deglución. } \\
\text { - Limitación de la elevación de la laringe. } \\
\text { - Movimientos incompletos del hueso hioides. } \\
\text { - Cierre incompleto de la epiglotis. } \\
\text { - Vestibulo de la laringe: } \\
\text { 1) penetración subepiglótica } \\
\text { 2) penetración supraglótica } \\
\text { 3) penetración/aspiración traqueal } \\
\text { - Inicio tardio de la deglución faríngea. } \\
\text { - Ausencia de deglución faríngea. }\end{array}$ \\
\hline FISIOLOGIA DE LA FASE ESOFAGICA & DISFUNCIÓN ESOFAGICA \\
\hline - Apertura del SFE. & $\begin{array}{l}\text { - Apertura incompleta o nula del SFE. } \\
\text { - Regurgitación desde el esófago hasta los senos piriformes. } \\
\text { - Peristalsis incompleta. } \\
\text { - Estenosis. }\end{array}$ \\
\hline
\end{tabular}

Fuente: (Quintana Sanjuás, Al Rifai, Carrero González, \& San Román Rodríguez, 2018)

Es decir, que la deglución puede alterarse por consecuencia neurológica, muscular, respiratoria o estructural atómica. Cuando sucede un compromiso del proceso deglutorio se hace alusión a la disfagia, nomenclatura diagnóstica que corresponde a la alteración de la alimentación(Vargas, Navas, \& Medina, 2016). Por eso, es importante poder comprender la disfagia. Se define como la sensación subjetiva de no progresión adecuada del bolo alimenticio con la deglución(Martín, Martín, \& Gurruchaga, 2005). 


\section{Diagnóstico diferencial de la disfagia}

Vol. 3, núm. 1., (2019)

Marcelo Kevin Taiano Espinoza; William Aarón Vargas Samerón; Luis Carlos, Sánchez López;

Paola Alexandra Bonifaz Aquino

Por lo tanto, esta investigación tiene como objetivo general analizar el diagnóstico diferencial de la disfagia. La metodología empleada se basó en investigaciones de tipo documental y bibliográfica.

\section{Método.}

Una de las partes más importantes en la problemática de la deglución es la disfagia por lo que se desarrolló un análisis de las características de esta enfermedad conjuntamente con el diagnóstico diferencial a través de la metodología de tipo documental y bibliográfica. Estas investigaciones se basaron en la ayuda de medios como textos, documentos y artículos científicos publicados disponibles en la web.

\section{Resultados.}

\section{Características de la disfagia}

Una de las enfermedades que puede ocurrir dentro de la deglución es la disfagia. El término disfagia proviene del griego y significa dificultad para comer, la cual se define como cualquier dificultad o anormalidad de la deglución(Martín Masot \& Ortega Páez, 2017). La disfagia es un síntoma que hace referencia a la dificultad o molestia para formar, mover y/o transportar el bolo alimenticio desde la boca al estómago(Quintana Sanjuás, Al Rifai, Carrero González, \& San Román Rodríguez, 2018).

Existe problemas cuando se confunde la disfagia dentro de otras enfermedades por su similitud y consecuencia, entre ellas está la odinofagia o sialorrea. Odinofagia se define como dolor al tragar, que también puede estar presente en la disfagia y la Sialorrea se define como 


\section{Diagnóstico diferencial de la disfagia}

Vol. 3, núm. 1., (2019)

Marcelo Kevin Taiano Espinoza; William Aarón Vargas Samerón; Luis Carlos, Sánchez López; Paola Alexandra Bonifaz Aquino

exceso de secreción de saliva, que babea y que puede acompañar a la disfagia orofaríngea(Martín Masot \& Ortega Páez, 2017).

Esto quiere decir que la disfagia es una alteración del funcionamiento del mecanismo de la deglución. Puede ocurrir en cualquiera de sus fases (oral, faríngea, esofágica), así como la posibilidad de que el alimento pueda ser dirigido de manera errónea a vías aéreas inferiores y se produzca aspiración del contenido alimenticio(Quintana Sanjuás, Hurtado Ruzza, ÁlvarezCalderón Iglesias, \& Patiño Castiñeira, 2018).Puede corresponder a causas de disfunciones orofaríngeas o esofágicas, a partir del punto de vista anatómico, estructurales o funcionales desde el punto de vista fisiopatológico (Cuello Del Pezo, 2017).Las causas de ladisfagia orofaríngea y de la disfagia esofágica se muestran en la Tabla 3.

La disfagia orofaríngea engloba las alteraciones de la deglución de origen oral, faríngeo, laríngeo y del esfínter esofágico superior y supone casi el $80 \%$ de las disfagias diagnosticadas. La disfagia esofágica se refiere a las alteraciones en el esófago superior, el cuerpo esofágico, el esfínter inferior y cardias, y generalmente es producida por causas mecánicas, y supone el 20\% de las disfagias que se diagnostican.(Velasco \& García-Peris, 2009, pág. 57) 


\section{Diagnóstico diferencial de la disfagia}

Vol. 3, núm. 1., (2019)

Marcelo Kevin Taiano Espinoza; William Aarón Vargas Samerón; Luis Carlos, Sánchez López;

Paola Alexandra Bonifaz Aquino

\section{Tabla 3. Causas de lade la disfagia orofaríngea y de la disfagia esofágica}

\begin{tabular}{|c|c|}
\hline Causas de disfagia orofaríngea & Causas de disfagia esofágica \\
\hline A) ORGÁNICAS: Obstrucción de la luz esofágica & A) ORGÁNICAS: Obstrucción mecánica \\
\hline a) Intrínsecas: & a) Intrínsecas: \\
\hline Anillos congénitos o adquiridos & Inflamatorias: esofagitis péptica, por reflujo, cáustica \\
\hline Síndrome Plummer-Vinson & Cuerpos extraños \\
\hline Procesos inflamatorios & Anillo de Schatzki \\
\hline Neoplasias orofaríngeas & Divertículos esofágicos \\
\hline Resecciones quirúrgicas & Neoplasias benignas y malignas \\
\hline Divertículo Zenker & b) Extrínsecas: \\
\hline b) Extrínsecas: & Adenopatías \\
\hline Hipertrofia tiroidea & Candiomegalia \\
\hline Hipertrofia paratiroidea & Aneurismas de aorta \\
\hline Adenopatías cervicales & Neoplasias mediastínicas y broncopulmonares \\
\hline Hipertrofia del timo & B) FUNCIONALES: Alteración neuromuscular \\
\hline Hiperostosis columna cervical & a) Sistema nervioso central: \\
\hline B) FUNCIONALES: Alteración neuromuscular & Accidente cerebrovascular \\
\hline a) Del sistema nervioso central: & Degenerativa \\
\hline Accidentes cerebrovasculares & Desmielinizante \\
\hline Parkinson, esclerosis múltiple & b) Neuropatía periférica: \\
\hline Esclerosis lateral amiotrófica & Diabética y alcohólica \\
\hline b) De la placa motora: & c) Placa motora: \\
\hline Miastenia gravis & Miastenia gravis \\
\hline c) Del sistema nervioso periférico: & d) Miopatías: \\
\hline Poliomielitis bulbar & Músculo esquelético: polimiositis y dermatomiositis \\
\hline Neuropatías: diabética y alcohólica & Músculo liso: esclerodermia \\
\hline d) Del músculo esquelético: & e) Esofágica: \\
\hline Polimiositis & Acalasia \\
\hline Dermatomiositis & Espasmo difuso \\
\hline Distrofias musculares & Enfermedad de Chagas \\
\hline Miopatías metabólicas & Reflujo gastroesofágico \\
\hline
\end{tabular}

Fuente: (Epprecht González, Trobat Company, \& Novas Valiente, 2006)

La disfagia se puede clasificar según su origen en disfagia motora y disfagia mecánica u obstructiva. La disfagia motora se debe a la alteración o incoordinación de los mecanismos de la deglución, y se puede producir tanto con la ingesta de líquidos como de sólidos (HUAYTA, 2018). La disfagia mecánicasobresale en todas las series la estenosis péptica benigna, consecuencia de un reflujo crónico del contenido ácido gástrico hacia el esófago que condiciona 


\section{Diagnóstico diferencial de la disfagia}

Vol. 3, núm. 1., (2019)

Marcelo Kevin Taiano Espinoza; William Aarón Vargas Samerón; Luis Carlos, Sánchez López; Paola Alexandra Bonifaz Aquino

la aparición de lesiones en la mucosa esofágica y estenosis posterior: la esofagitis péptica(Martín, Martín, \& Gurruchaga, 2005).

Este tipo de enfermedad puede aparecer en personas de la tercera edad la cual puede disminuir la calidad de vida, como también que es consecuencia de tratamientos para neoplasias en el tracto digestivo. Se ha reportado una prevalencia de disfagia entre el 8.4 y el $16 \%$ de la población general, que aumenta con la edad, llegando a cifras hasta del $26 \%$ para personas mayores de 76 años (Gutiérrez, Ruales Suárez, Cadavid, Fernando, \& Rengifo Varona, 2015). En la Tabla 4 se puede observar como las causas más frecuentes de disfagia en la población mayor.

\section{Tabla 4. Causas más frecuentes de disfagia en la población mayor}

\begin{tabular}{lll} 
Localización disfagia & Lesiones estructurales & Enfermedades neuromusculares \\
\hline I. Disfagia orofaríngea & Intrínsecas: & S.N.C. \\
& Tumores, estenosis, cirugía, & ACVA, demencias, Parkinson, \\
& inflamatorias, infecciosas. & esclerosis lateral amiotrófica. \\
& Extrínsecas: & Nervios craneales: \\
& Bocio, vasculares, vertebrales. & Diabetes mellitus, parálisis laríngeo. \\
& & Músculo: \\
& & Miopatías, distrofias, hipo/hipertiroidismo, \\
& Intrínsecas: & amiloidosis, disfunción cricofaríngea. \\
\hline II. Disfagia esofágica & Neoplasias, estenosis, cirugía, & Carcinoma esofágico. \\
& cáusticas, cuerpos extraños. & Estenosis péptica. \\
& Extrínsecas: & Compresión vascular (aneurisma de aorta). \\
& Tumores, aneurisma, cardiomegalia. & Adenopatías mediastínicas (carcinoma \\
& & de pulmón, linfoma, tuberculosis).
\end{tabular}

Fuente: (Jiménez Rojas, Corregidor Sánchez, \& Gutiérrez Bezón, 2018)

Ahora, para la población en general existen dos complicaciones de importancia que puede generar la disfagia. 


\section{Diagnóstico diferencial de la disfagia}

Vol. 3, núm. 1., (2019)

Marcelo Kevin Taiano Espinoza; William Aarón Vargas Samerón; Luis Carlos, Sánchez López;

Paola Alexandra Bonifaz Aquino

- Malnutrición y deshidratación, al generar disminución de la eficacia de la deglución.

- Penetración o aspiración, al generar disminución de la seguridad de la deglución. Se denomina penetración la entrada de contenido orofaríngeo en el vestíbulo laríngeo sin rebasar las cuerdas vocales, cuando las sobrepasa se denomina aspiración.(Ossa Echevarría, Castro Macía, \& VV., 2018, pág. 21)

La sospecha de disfagia debe plantearse en pacientes que refieren síntomas aparentemente diversos, el paciente que tose o se atraganta al comer muy probablemente está teniendo una aspiración (Velasco \& García-Peris, 2009). En la Tabla 5 muestran los síntomas que están orientados a la disfagia y sus posibles causas.

Tabla 5. Síntomas que están orientados a la disfagia

\begin{tabular}{ll}
\hline Síntomas objetivados & Posible causa \\
Babeo & Parálisis facial \\
Falta de propulsión del bolo & Trastorno de las praxias linguales \\
Estornudos, regurgitación nasal, mal olor & Insuficiencia velofaríngea \\
Residuos en boca después de deglución & Trastorno de praxias linguales \\
Sensación de cuerpo extraño, carraspera & Reflujo extraesofágico \\
Vómitos & Falta de apertura EES \\
Mal aliento, regurgitación tardía de alimentos & Divertículo faringoesofágico \\
Gianosis y atragantamiento con deglución & Aspiración \\
Tos con deglución & Aspiración \\
Voz húmeda & Penetración laríngea \\
Neumonía por aspiración & Aspiración \\
\hline
\end{tabular}

Fuente: (Epprecht González, Trobat Company, \& Novas Valiente, 2006)

Lo importante es el manejo y control del paciente el cual debe ser interdisciplinario. Además del laringólogo, deben incorporarse todos los recursos humanos y técnicos para 


\section{Diagnóstico diferencial de la disfagia}

Vol. 3, núm. 1., (2019)

Marcelo Kevin Taiano Espinoza; William Aarón Vargas Samerón; Luis Carlos, Sánchez López;

Paola Alexandra Bonifaz Aquino

realizarlo de forma efectiva, fundamentalmente logopedas, especialistas en nutrición, enfermería, y la colaboración de especialistas en vía digestiva, todos con entrenamiento específico en la valoración y tratamiento de la disfagia (Cabrera Morín \& Rico Cantillo, 2018).

En el Ecuador, específicamente en la ciudad de Cuenca, se han realizado estudios sobre la disfagia en los adultos mayores en los principales geriátricos de la localidad, como se detalla en la Tabla 6. De aquí, se obtuvo una relación de la disfagia con los factores de riesgo la cual refleja que la mayoría presentaron factores metabólicos la cual no es causa directa, al contrario, se puede considerar como un factor predisponente a la presencia de un riesgo neurológico. Esto se muestra en la Tabla 7

Tabla 6. Matriz de centros geriátricos de la ciudad de Cuenca, Ecuador

\begin{tabular}{lcc}
\hline \multicolumn{1}{c}{ Nombre de Centro geriátrico } & Zona & $\begin{array}{c}\text { No. de adultos } \\
\text { mayores }\end{array}$ \\
\hline Centro Gerontológico "María Reina de la Paz" & Urbana & 72 \\
Casa Hogar Miguel León. & Urbana & 56 \\
Fundación Gerontológica Plenitud. & Urbana & 16 \\
Residencia Geriátrica "San Andrés”. & Urbana & 18 \\
\hline
\end{tabular}

Total: 162

Fuente: (Carrillo Erique \& Gárate Rugel, 2018) 


\section{Diagnóstico diferencial de la disfagia}

Vol. 3, núm. 1., (2019)

Marcelo Kevin Taiano Espinoza; William Aarón Vargas Samerón; Luis Carlos, Sánchez López;

Paola Alexandra Bonifaz Aquino

Tabla 7. Distribución según factores de riesgo en relación al grado de disfagia de adultos

mayores evaluados en los centros geriátricos urbanos de Cuenca, Ecuador

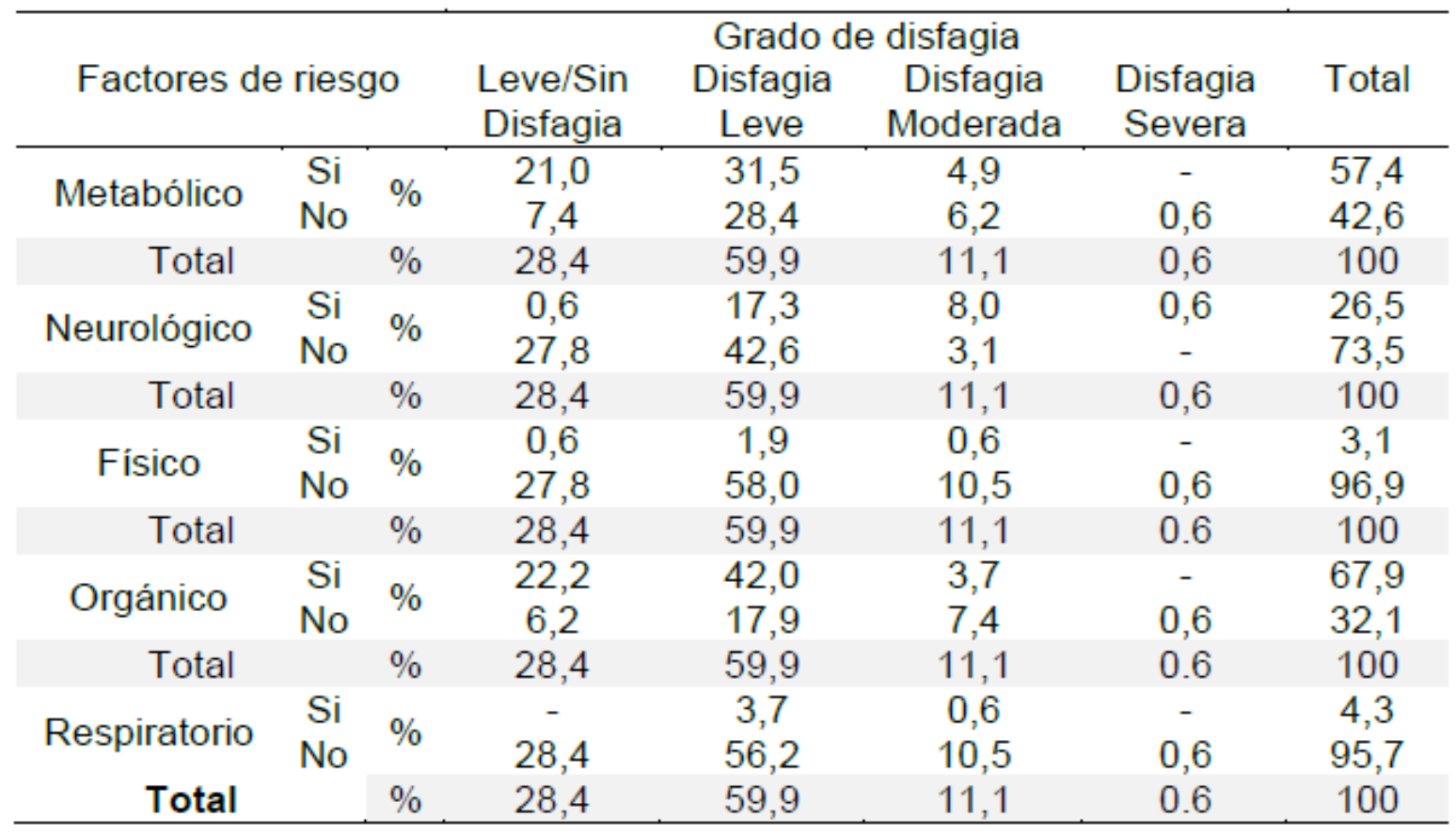

Fuente: (Carrillo Erique \& Gárate Rugel, 2018)

Antes de realizar cualquier diagnostico se debe realizar un estudio personal al paciente para poder confrontar los síntomas y así poder obtener una mejor percepción de sufrir disfagia. Esto se puede hacer a través de un test clínico para el diagnóstico de disfagia orofaríngea como se muestra en la Tabla 8. 


\section{Diagnóstico diferencial de la disfagia}

Vol. 3, núm. 1., (2019)

Marcelo Kevin Taiano Espinoza; William Aarón Vargas Samerón; Luis Carlos, Sánchez López; Paola Alexandra Bonifaz Aquino

\section{Tabla 8. Test clínico para el diagnóstico de disfagia orofaríngea}

1. ¿Cuanta dificultad tiene actualmente para tragar?

Leve $\square \quad$ Moderada $\square \quad$ Severa $\square$

2. ¿Cuanta dificultad tiene para tragar líquidos como el agua, té, bebidas calientes?

Leve $\square \quad$ Moderada $\square \quad$ Severa $\square$

2b. ¿Tose o se atraganta?

Nunca $\square \quad$ A veces $\square \quad$ Siempre

3. ¿Cuanta dificultad tiene para tragar líquidos espesos como sopas, natillas, batidos?

Leve $\square \quad$ Moderada $\square \quad$ Severa $\square$

3b. ¿Tose o se atraganta?

Nunca $\square \quad$ A veces $\square \quad$ Siempre $\square$

4. ¿Cuanta dificultad tiene para tragar comidas blandas como puré, tortilla?

Leve $\square \quad$ Moderada $\square \quad$ Severa $\square$

4b. ¿Tose o se atraganta?

Nunca $\square \quad$ A veces $\square \quad$ Siempre $\square$

5. ¿Cuanta dificultad tiene para tragar comidas sólidas como pan, carne, fruta?

Leve $\square \quad$ Moderada $\square \quad$ Severa $\square$

5b. ¿Tose o se atraganta?

Nunca $\square \quad$ A veces $\square \quad$ Siempre $\square$

6. ¿Tiene alguna dificultad para tratar su propia saliva?

Leve $\square \quad$ Moderada $\square \quad$ Severa $\square$

7. ¿Necesita tragar más de una vez para tragar lo que tiene en la boca?

Nunca $\square \quad$ A veces $\square \quad$ Siempre $\square$

8. Al tragar ¿presenta dolor en la garganta?

Nunca $\square \quad$ A veces $\square \quad$ Siempre $\square$

9. ¿Presenta resfriados comunes?

Nunca $\square \quad$ A veces $\square \quad$ Siempre $\square$
10. Alguna vez fumó o fuma?
Sí $\square$
No $\square$

11. ¿Cómo valoraría hoy la severidad de su problema de deglución?

Leve $\square \quad$ Moderada $\square \quad$ Severa $\square$

\section{TEST DE CALIDAD DE VIDA}

1. ¿En general, ud. piensa que comer debería ser una experiencia agradable?
Sí [
No $\square$
No sé $\square$

2. $¿$ Ud. cree que comer ocupa una parte agradable de su día?

Sí $\square \quad$ No $\square \quad$ Nosé $\square$

3. Si la respuesta es no, $i$ es debido por su dificultad para tragar?
Sí $\square$
No $\square$
No sé

4. ¿Ud. se siente agobiado por sus problemas al tragar en las comidas?
Sí
No $\square$
No sé $\square$

5. Si la respuesta es sí (en la pregunta n. ${ }^{\circ}$ ) ¿ ¿ud. evita comer con otras personas debido a dichos problemas?
Sí
No $\square$
No sé

6. Si la respuesta es sí (en la pregunta n. ${ }^{\circ}$ ) ¿ud. se siente incómodo debido a esta condición en las comidas?
Sí
No $\square$
No sé

7. Si la respuesta es sí (en la pregunta n.ำ ¿ud. se siente ansioso o temeroso durante las comidas?
Sí []
No $\square$
No sé $\square$

8. ¿Ud. cree que sus problemas al tragar hacen su vida menos agradable?
No $\square$
No sé

Fuente: (Velasco \& García-Peris, 2009)

Con la información obtenida se puede proceder a realizar un diagnóstico que se puede manifestar como un algoritmo que se muestra en la Figura 7. 


\section{Diagnóstico diferencial de la disfagia}

Vol. 3, núm. 1., (2019)

Marcelo Kevin Taiano Espinoza; William Aarón Vargas Samerón; Luis Carlos, Sánchez López;

Paola Alexandra Bonifaz Aquino

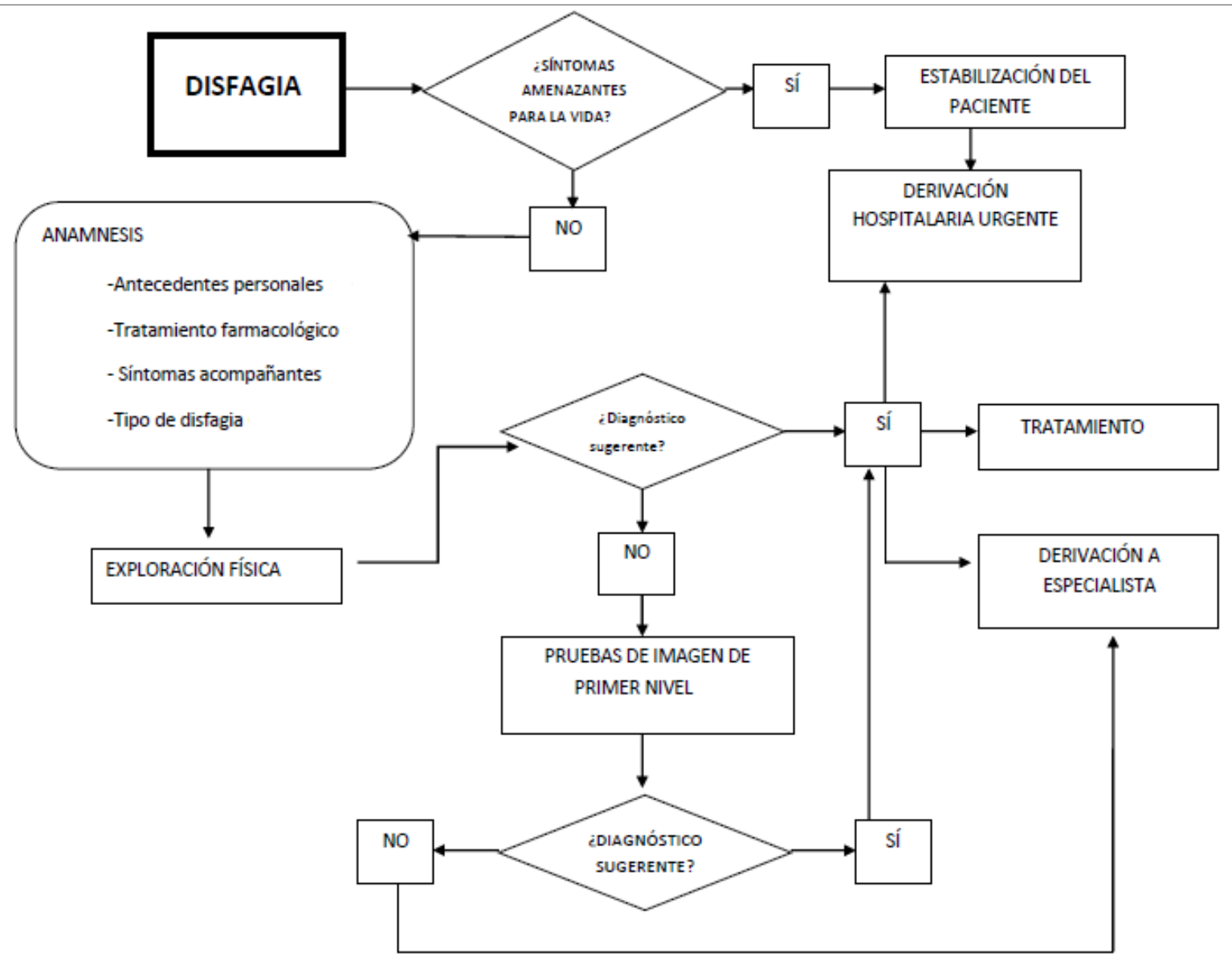

Figura 7. Algoritmo para el diagnóstico de la disfagia

Fuente: (Martín Masot \& Ortega Páez, 2017)

Seguidamente se puede realizar un examen físico que permitirá deducir, más claramente, la patología de la disfagia. Dentro de este examen se recurre a la inspección visual, táctil y sonara de los síntomas que puedan provenir de la disfagia.

Se analiza la postura y tono muscular, la capacidad para mantenerse erguido durante las comidas, sentado y en una posición adecuada. Se valora las alteraciones en la voz, si es aérea, tensa, nasal o húmeda, siendo ésta última un probable signo de penetración, así como también 


\section{Diagnóstico diferencial de la disfagia}

Vol. 3, núm. 1., (2019)

Marcelo Kevin Taiano Espinoza; William Aarón Vargas Samerón; Luis Carlos, Sánchez López; Paola Alexandra Bonifaz Aquino

son de interés los carraspeos frecuentes o la tos seca. Se realiza exploración orofacial. La alteración del sello labial produce babeo; su funcionalidad se constata ordenando fruncir los labios, silbar, inflar alternativamente los carrillos o decir el fonema /p/. También se evalúa los movimientos mandibulares. Se explora el tono de la lengua, así como su capacidad de movimiento, el fonema /t/ y la capacidad de hacer chasquidos linguales. La valoración del sello palatogloso, cuya incompetencia puede producir regurgitación nasal y aspiración predeglutoria; para comprobar su funcionalidad es útil el fonema /k/ y la evaluación de la nasalidad. Se realiza la palpación cervical, donde se deben descartar masas, tumoraciones o adenopatías, descartar aumento de volumen de la glándula tiroides y la presencia de nódulos tiroideos. (Cabrera Morín \& Rico Cantillo, 2018, págs. 55-56)

\section{Diagnóstico diferencial}

Para poder reflejar un tratamiento especial para una enfermedad es necesario el análisis y comprensión de los síntomas manifestados técnicamente a través de evaluaciones médicas. Es aquí donde surgen los diagnósticos. El diagnóstico es un proceso inferencial, realizado a partir de un "cuadro clínico", destinado a definir la enfermedad que afecta a un paciente(Herrera Galiano, 2011).

Al hablar de "cuadro clínico", hay 3 elementos que se interrelacionan y que vale la pena definir. El síndrome o conjunto de síntomas y signos que presenta el paciente a raíz de la enfermedad; la enfermedad, tal cual lo leemos en textos de medicina, por ejemplo, insuficiencia suprarrenal, cáncer del pulmón, hipertensión arterial; y el contexto, que es el 


\section{Diagnóstico diferencial de la disfagia}

Vol. 3, núm. 1., (2019)

Marcelo Kevin Taiano Espinoza; William Aarón Vargas Samerón; Luis Carlos, Sánchez López;

Paola Alexandra Bonifaz Aquino

ambiente social, económico, psicológico en que se sitúa la persona que padece la enfermedad.(Capurro \& Rada, 2007, pág. 534)

El diagnóstico requiere intuición y a su vez un análisis objetivo de la enfermedad que se padece. El proceso diagnóstico requiere tanto de razonamiento lógico como de habilidad para el reconocimiento de patrones, lo cual permite un correcto y eficiente diagnóstico para prevenir, curar y focalizar el tratamiento de las enfermedades en nuestros pacientes(Vera, Letelier, María, \& Carvajal, 2005).

Existe un proceso que sirve para descartar diagnóstico basado en la confirmación de las certezas e intuición del médico el cual es llamado umbrales de decisión. Cuando no es necesario realizar nuevos test, sino iniciar el manejo concordante con el diagnóstico se llama umbral terapéutico y cuando se deja de requerir nuevos test donde la probabilidad es tan baja que no se justifica se llama umbral de estudio adicional (Capurro \& Rada, 2007). Esto se puede observar en la Figura 8.

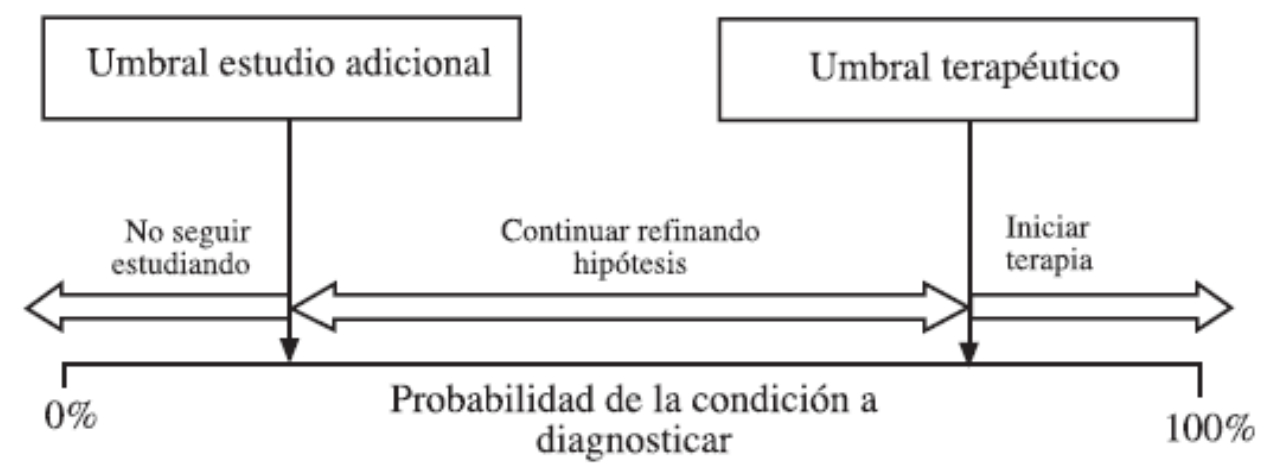

Figura 8. Umbrales de decisión

Fuente: (Capurro \& Rada, 2007) 


\section{Diagnóstico diferencial de la disfagia}

Vol. 3, núm. 1., (2019)

Marcelo Kevin Taiano Espinoza; William Aarón Vargas Samerón; Luis Carlos, Sánchez López; Paola Alexandra Bonifaz Aquino

Ahora realizar un diagnóstico diferencial es muy importante ya que puede eliminar aquellas patologías dentro de la enfermedad planteada y deja solo la alternativa que justifique el cuadro clínico del paciente. Se trata, pues, de excluir entidades que pueden confundirse con el cuadro del paciente, por lo que es quizás la parte más importante de la discusión diagnóstica(Herrera Galiano, 2011).Es muy importante establecer inicialmente una exclusión de cualquier patología orgánica que pudiera producir los síntomas(De Lucas-Taracena \& MontañesRada, 2006).

Es por ello que el médico debe tener una organización de los posibles diagnósticos para poder hacer el diagnóstico diferencial con el fin de seleccionar la mejor posibilidad diagnóstica, a través de:

a) Enumerar todas las posibilidades, según órganos o sistemas, para descartarlas en forma simultánea con una serie de exámenes; corresponde a una forma de posible de aproximación.

b) Ordenar estas posibilidades diagnósticas de acuerdo a su probabilidad relativa; corresponden a una forma probabilística de aproximación.

c) Estudiar las posibilidades diagnósticas según su pronóstico (descartar o confirmar primero la posibilidad de peor pronóstico).

d) Evaluar las posibilidades diagnósticas según un criterio pragmático (diagnosticar las que respondan mejor al tratamiento).(Vera, Letelier, María, \& Carvajal, 2005, págs. 196-197) 


\section{Diagnóstico diferencial de la disfagia}

Vol. 3, núm. 1., (2019)

Marcelo Kevin Taiano Espinoza; William Aarón Vargas Samerón; Luis Carlos, Sánchez López;

Paola Alexandra Bonifaz Aquino

Uno de los mecanismos para poder eliminar posibles diagnostico no favorables es el conocimiento de la historia clínica del paciente. Esto es antecedentes patológicos, operaciones anteriores, alergia a medicamentos, elementos epidemiológicos, ocupación, sexo, raza, interrogatorio por aparatos, síntomas que son patognomónicos de algunas entidades, elementos negativos, etcétera, que puede ayudar en la eliminación de algunas afecciones(Herrera Galiano, 2011).

Para el desarrollo de un diagnóstico diferencial de la disfagiaes necesario el implemento del test, el examen físico y concomimiento de la historia clínica del paciente. Aunado también al conocimiento de los síntomas de esta enfermedad. En la Figura 9 se puede observar el diagnóstico diferencial de la disfagia.

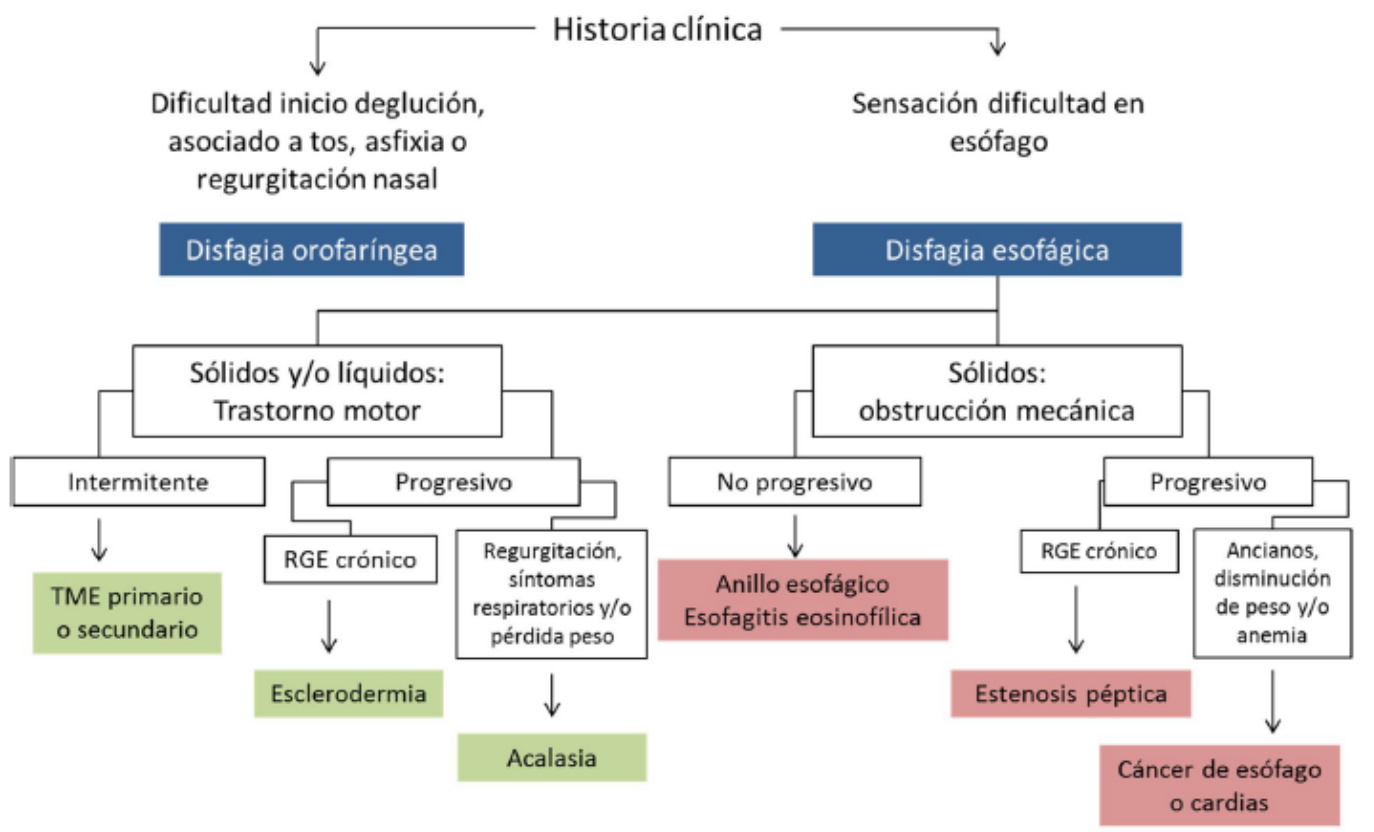

Figura 9. Algoritmo del diagnóstico diferencial de la disfagia

Fuente: (Ceballos, 2015) 


\section{Diagnóstico diferencial de la disfagia}

Vol. 3, núm. 1., (2019)

Marcelo Kevin Taiano Espinoza; William Aarón Vargas Samerón; Luis Carlos, Sánchez López; Paola Alexandra Bonifaz Aquino

Después de la determinación en el diagnóstico diferencial se aplica el tratamiento médico que permita la mejora de la calidad de vida. Este tratamiento es individualizado y ejecutado por un grupo multidisciplinario. Los objetivos nutricionales se deben establecer de inmediato donde la nutrición requerirá la colocación de sondas nasogástricas o gastrostomías temporales o permanentes; y para los casos graves de excesiva secreción de saliva puede ser necesario el tratamiento quirúrgico de las secreciones.

\section{Discusión y conclusiones.}

Uno de los objetivos de poder establecer un equilibrio bioquímico y físico del organismo humano es mantener una alimentación saludable. Por lo cual, la deglución cumple con ese objetivo fundamental. Es un proceso neuromuscular en la cual los alimentos pasan de la boca al estómago para que luego sean procesados química y físicamente. Este proceso complejo pasa por varias etapas conocidas como la oral faríngea y esofágica. Algún problema en estas etapas de deglución y entonces se empieza a romper el equilibrio y hacen aparecer ciertas lesiones que se transforman en enfermedades. Una de estas consecuencias de la alteración o lesión en algunos miembros que conforman la tarea de la deglución es la disfagia.

La disfagia es un problema con la transportación del bolo alimenticio de la boca hacia el estómago, es decir se presenta una dificultad de mover, formar y transportar el alimento. Este problema ocurre en alguna parte de las fases de la deglución como es la oral, faríngea y esofágica. Este problema puede estar dividido en dos grandes grupos en la disfagia orofaríngea y la disfagia esofágica, donde la primera se presenta en mayor cantidad de veces en la población que padece esta enfermedad. Aunado a esto la población de edad mayor son los que sufren 


\section{Diagnóstico diferencial de la disfagia}

Vol. 3, núm. 1., (2019)

Marcelo Kevin Taiano Espinoza; William Aarón Vargas Samerón; Luis Carlos, Sánchez López;

Paola Alexandra Bonifaz Aquino

mayormente esta enfermedad debido a que a medida que se avanza en el tiempo algunos de los órganos pueden empezar a perder la vitalidad. En el Ecuador, la gran mayoría de las personas de la tercera edad sufren esta consecuencia a través de factores metabólicos de origen neurológicos.

Al conocer los síntomas y las causa por las cuales se padece de disfagia, una de las principales herramientas en la elaboración del historial clínico y con el fin de empezar a diagnosticar la enfermedad es el test clínico. Está basado en preguntas relacionadas a la enfermedad con el objetivo de poder descartar posibles errores en diagnósticos. Para ello, se realiza el diagnóstico diferencial es vital para obtener un tratamiento adecuado. Consiste en ir descartando entidades que confundan con la ayuda del historial clínico. Por lo tanto, determinar el diagnóstico diferencial de la disfagia se requiere del historial del paciente, del test clínico que ayudara a conocer los síntomas que padece y del examen físico que se realiza en primer momento.

\section{Bibliografía.}

Cabrera Morín, P., \& Rico Cantillo, P. (2018). EVALUACIÓN CLÍNICA Y EXPLORACIÓN EN LA DISFAGIA. En M. Rodríguez Acevedo, P. Vaamonde Lago, T. González Paz, A. Quintana Sanjuás, \& M. González Cortés, DISFAGIA OROFARÍNGEA: ACTUALIZACIÓN Y MANEJO EN POBLACIONES ESPECÍFICAS (págs. 53-58). España: Sociedad Gallega de Otorrinolaringología y Patología Cérvico-Facial.

Capurro, D., \& Rada, G. (2007). El proceso diagnóstico. Revista médica de Chile, 135(4), 534538.

Carrillo Erique, C. E., \& Gárate Rugel, D. C. (2018). Incidencia de disfagia en el adulto mayor de los centros geriátricos urbanos de Cuenca 2017-2018. Cuenca - Ecuador: Trabajo de Grado - Universidad de Cuenca.

Ceballos, F. L. (2015). Epidemiología de la disfagia en la población española. Madrid - España: Trabajo de Grado - Universidad Complutense de Madrid. 


\section{Diagnóstico diferencial de la disfagia}

Vol. 3, núm. 1., (2019)

Marcelo Kevin Taiano Espinoza; William Aarón Vargas Samerón; Luis Carlos, Sánchez López;

Paola Alexandra Bonifaz Aquino

Cuello Del Pezo, F. A. (2017). Proceso de atención de enfermería en paciente adulto mayor con disfagia según el modelo de conservación de Myra Levine. Machala - Ecuador: Trabajo de Grado - Universidad Técnica de Machala.

De Lucas-Taracena, M. T., \& Montañes-Rada, F. (2006). Fobia a tragar: clínica, diagnóstico y tratamiento. Actas Esp Psiquiatr, 34(5), 309-316.

Epprecht González, P., Trobat Company, F., \& Novas Valiente, E. (2006). Fisiopatología de la deglución y aspiración. En M. Quer Agustí, SUPLEMENTOS DE ACTUALIZACIÓN EN ORL (págs. 65-69). Madrid - España: SANIDAD EDICIONES.

Gutiérrez, A., Ruales Suárez, K., Cadavid, G., Fernando, L., \& Rengifo Varona, M. L. (2015). Escalas de calidad de vida y valoración de los síntomas en disfagia. Revista Med, 23(1), $50-55$.

Herrera Galiano, A. \&. (2011). El proceso diagnóstico y su enseñanza en la medicina. Revista Habanera de Ciencias Médicas, 10(1), 126-134.

HUAYTA, F. (2018). DISFAGIA EN EL TRATAMIENTO DE UN CENTRO PSIQUIÁTRICO. Trabajo de Grado - UNIVERSIDAD COMPLUTENSE.

Jiménez Rojas, C., Corregidor Sánchez, A., \& Gutiérrez Bezón, C. (2018). DISFAGIA. Tratado de geratría para residentes. Capítulo 53, 545-553.

Martín Masot, R., \& Ortega Páez, E. (2017). Guía de Algoritmos en Pediatría de Atención Primaria. Disfagia en el paciente pediátrico. Obtenido de algoritmos.aepap.org

Martín, M. G., Martín, F. A., \& Gurruchaga, F. R. (2005). Disfagia en adolescentes: una causa poco valorada. VOX PAEDIATRICA, 13, 2, 42-47.

Ossa Echevarría, C., Castro Macía, O., \& VV., M. V. (2018). ALTERACIONES DE LA SEGURIDAD EN DISFAGIA OROFARÍNGEA: ASPIRACIÓN. En M. Rodríguez Acevedo, P. Vaamonde Lago, T. González Paz, A. Quintana Sanjuás, \& M. González Cortés, DISFAGIA OROFARÍNGEA: ACTUALIZACIÓN Y MANEJO EN POBLACIONES ESPECÍFICAS (págs. 21-26). España: Sociedad Gallega de Otorrinolaringología y Patología Cérvico-Facial.

Quintana Sanjuás, A., Al Rifai, M., Carrero González, A., \& San Román Rodríguez, E. (2018). FISIOPATOLOGÍA DE LA DEGLUCIÓN. En M. Rodríguez Acevedo, P. Vaamonde Lago, T. González Paz, A. Quintana Sanjuás, \& M. González Cortés, DISFAGIA OROFARÍNGEA: ACTUALIZACIÓN Y MANEJO EN POBLACIONES ESPECÍFICAS. (págs. 15-20). España: Sociedad Gallega de Otorrinolaringología y Patología CérvicoFacial.

Quintana Sanjuás, A., Hurtado Ruzza, R. A., Álvarez-Calderón Iglesias, O., \& Patiño Castiñeira, B. M. (2018). DISFAGIA OROFARÍNGEA: DEFINICIÓN, EPIDEMIOLOGÍA Y 


\section{Diagnóstico diferencial de la disfagia}

Vol. 3, núm. 1., (2019)

Marcelo Kevin Taiano Espinoza; William Aarón Vargas Samerón; Luis Carlos, Sánchez López;

Paola Alexandra Bonifaz Aquino

CALIDAD DE VIDA. En M. Rodríguez Acevedo, P. Vaamonde Lago, T. González Paz, A. Quintana Sanjuás, \& M. González Cortés, DISFAGIA OROFARÍNGEA: ACTUALIZACIÓN Y MANEJO EN OROFARÍNGEA: DEFINICIÓN, EPIDEMIOLOGÍA Y CALIDAD DE VIDA. DISFAGIA OROFARÍNGEA, 1. (págs. 1-8). España: Sociedad Gallega de Otorrinolaringología y Patología Cérvico-Facial.

Vargas, K. S., Navas, C. M., \& Medina, T. (2016). Evaluación fonoaudiológica de la disfagia: encuentros y disonancias. Revista Colombiana de Rehabilitación, 15(1), 22-31.

Velasco, M., \& García-Peris, P. (2009). Causas y diagnóstico de la disfagia. Nutrición Hospitalaria, 2(2), 56-65.

Vera, P. G., Letelier, S., María, L., \& Carvajal, C. (2005). Guía para el análisis crítico de estudios que evalún exámenes diagnósticos. Revista chilena de obstetricia y ginecología, 70(3), 196-202.

Vilardell Navarro, N. (2017). Fisiopatología, historia natural, complicaciones y tratamiento de la disfagia orofaríngea asociada al ictus. Barcelona - España: Trabajo de Grado Universidad Autónoma de Barcelona. 